\title{
Multivariate Spectral Estimation based on the concept of Optimal Prediction
}

\author{
Mattia Zorzi
}

\begin{abstract}
In this technical note, we deal with a spectrum approximation problem arising in THREE-like multivariate spectral estimation approaches. The solution to the problem minimizes a suitable divergence index with respect to an a priori spectral density. We derive a new divergence family between multivariate spectral densities which takes root in the prediction theory. Under mild assumptions on the a priori spectral density, the approximation problem, based on this new divergence family, admits a family of solutions. Moreover, an upper bound on the complexity degree of these solutions is provided.
\end{abstract}

\section{Index Terms}

Generalized covariance extension problem, Spectrum approximation problem, Divergence family, Prediction theory, Convex optimization.

\section{INTRODUCTION}

The growing interest in the last years on the THREE-like multivariate spectral estimation approach, see the pioneering work [3], is certainly due to its appealing features. First, it leads to a convex optimization problem whose solution is a spectral density typically having an upper bound on the complexity degree. Second, the numerical solution is computed by efficient algorithms whose global convergence is guaranteed. Third, such approach allows a wide degree of freedom,

This note presents research results of the Belgian Network DYSCO (Dynamical Systems, Control, and Optimization), funded by the Interuniversity Attraction Poles Programme, initiated by the Belgian State, Science Policy Office. The scientific responsibility rests with its authors. This research is also supported by FNRS (Belgian Fund for Scientific Research).

M. Zorzi is with the Dipartimento di Ingegneria dell'Informazione, Università di Padova, via Gradenigo 6/B, 35131 Padova, Italy, zorzimatedei.unipd.it 
through the design of a bank of filters, in order to allow an higher resolution of the solution in the frequency bands of interest.

A THREE-like method may be outlined as follows. A finite length sequence extracted from a realization of a stochastic process, say $y$, is fed to a bank of filters. The output covariance is then used to extract information on the process by considering the family of spectral densities matching such output covariance. Therefore, the estimate of the spectral density of $y$ is chosen in this family. This task is accomplished by solving a spectrum approximation problem whose solution minimizes a divergence index with respect to an a priori spectral density.

It is clear that the solution highly depends on the divergence index. Many divergence indexes has been proposed in the last decade, [10], [9], [5], [4], [18], [19]. In particular, in [18] a multivariate extension to the Beta divergence family, [2], has been introduced. Making additional assumptions on the a priori spectral density besides bounded McMillan degree, it is possible to show that the corresponding spectrum approximation problem leads to a family of solutions. Moreover, such a family of solutions connects the ones obtained with the multivariate ItakuraSaito distance, [4], and the multivariate Kullback-Leibler divergence, [9]. It is worth noting that the (scalar) Itakura-Saito distance has been derived from the maximum likelihood of speech spectral densities, [11], whereas the (scalar) Kullback-Leibler divergence has been introduced to measure the difference between two probability distributions, [14]. Then, in [19] the family of solutions obtained by using the scalar Alpha divergence family, [1], has been considered. This family connects the solutions obtained by using the Kullback-Leibler divergence [9], [10]. Here, it is only required that the a priori spectral density has bounded McMillan degree. However, such a result cannot be extended to the multichannel case.

The main result of this note is the definition of a new multivariate divergence family which compares two spectral densities in the context of optimal prediction. This family also includes the multivariate Itakura-Saito distance. It is interesting to note this divergence family is not even known in the scalar case and it can be derived from the Alpha and the Beta divergence family. The corresponding spectrum approximation is then tackled. It turns out that it is possible to characterize a family of solutions to the problem with bounded McMillan degree by only making the mild assumption that the a priori spectral density has bounded McMillan degree as well. Finally, we face the feasibility issue for the spectrum approximation problem. Indeed, since the output covariance is estimated from the data, the family of spectral densities matching the 
(estimated) output covariance is typically empty. Hence, we approximate the output covariance, by solving a state covariance estimation problem, in such a way that the spectrum approximation problem is feasible.

The outline of the technical note is as follows. In Section $\amalg$ we introduce the new multivariate divergence family and in Section III the corresponding spectrum approximation problem. In Section IV we deal with the state covariance estimation problem. In Section $\mathrm{V}$ a simulation study, showing the features of the family of solutions, is then provided.

Finally, we introduce some convention and notation which will be used throughout the note: integration is on the unit circle with respect to the normalized Lebesgue measure. A star denotes transposition plus conjugation, $\mathcal{Q}_{n} \subset \mathbb{R}^{n \times n}$ denotes the $n(n+1) / 2$-dimensional real space of $n$-dimensional symmetric matrices and $\mathcal{Q}_{n,+}$ denotes the corresponding cone of positive definite matrices.

\section{A DIVERGENCE FAMILY IN THE CONTEXT OF OPTIMAL PREDICTION}

In this section we introduce a new divergence family for comparing two spectral densities which takes root in the prediction theory. Let $y=\left\{y_{k} ; k \in \mathbb{Z}\right\}$ be a stationary, full-rank, purely non-deterministic, zero mean, $\mathbb{R}^{m}$-valued gaussian process completely characterized by the spectral density $\Phi\left(\mathrm{e}^{j \vartheta}\right) \in \mathcal{S}_{+}^{m}(\mathbb{T})$, where $\mathcal{S}_{+}^{m}(\mathbb{T})$ denotes the family of coercive, bounded, $\mathbb{C}^{m \times m}$-valued spectral density functions on the unit circle $\mathbb{T}$. When $\Phi\left(\mathrm{e}^{j \vartheta}\right)$ is not known, we fix an a priori spectral density $\Psi\left(\mathrm{e}^{j \vartheta}\right) \in \mathcal{S}_{+}^{m}(\mathbb{T})$ describing $y$. Let $\hat{y}_{k}$ be the least-square linear one-stepahead predictor based on $\Psi$, and $e_{k}:=y_{k}-\hat{y}_{k}$ the corresponding innovation process. Accordingly $e_{k}^{N}:=L_{\Omega}^{-1}\left(y_{k}-\hat{y}_{k}\right)$ represents the normalized innovation process, with $L_{\Omega} L_{\Omega}^{T}=\mathbb{E}\left[e_{k} e_{k}^{T}\right]$. Since $\Psi \in \mathcal{S}_{+}^{m}(\mathbb{T})$, it admits a unique canonical left spectral factor such that

$$
\Psi\left(\mathrm{e}^{j \vartheta}\right)=W_{\Psi}\left(\mathrm{e}^{j \vartheta}\right) W_{\Psi}\left(\mathrm{e}^{j \vartheta}\right)^{*}
$$

with $W_{\Psi}(z) \in \mathrm{H}_{2}^{m \times m}(\mathbb{D})$, $\operatorname{det} W_{\Phi}(z) \neq 0$ in $\mathbb{D}:=\{z:|z| \geq 1\}$, and $W_{\Psi}(\infty)=L_{\Omega}$. Here, $\mathbb{H}_{2}^{m \times m}(\mathbb{D})$ denotes the Hardy space of analytic functions in $\mathbb{D}$ with square integrable radial limits. Thus, the normalized innovation process $e_{k}^{N}$ is obtained by filtering $y$ through the whitening filter, say $W_{\Psi}^{-1}(z),[13]$ :

$$
e_{k}^{N}=\sum_{l=-\infty}^{k} a_{\Psi}(k-l) y_{l},
$$


with

$$
W_{\Psi}^{-1}(z)=\sum_{l=0}^{\infty} a_{\Psi}(l) z^{-l}, \quad z \in \mathbb{D} .
$$

The spectral density of the normalized innovation process is

$$
E^{N}\left(\mathrm{e}^{j \vartheta}\right)=W_{\Psi}\left(\mathrm{e}^{j \vartheta}\right)^{-1} \Phi\left(\mathrm{e}^{j \vartheta}\right) W_{\Psi}\left(\mathrm{e}^{j \vartheta}\right)^{-*} .
$$

Clearly, if the a priori spectral density $\Psi$ coincides with the true spectral density $\Phi$, we have $E^{N}\left(\mathrm{e}^{j \vartheta}\right)=I$, i.e. the normalized innovation process is white gaussian noise (WGN) with zero mean and variance $I$. Therefore, $E^{N}$ represents a mismatch criterium which naturally occurs in prediction error estimation, [15], [16].

This lead us, as suggested in [12], to measure the mismatch between the true spectral density $\Phi$ and the a priori spectral density $\Psi$ by quantifying the mismatch between $E^{N}$ and $I$ :

$$
\mathcal{S}(\Phi \| \Psi)=\int \mathcal{F}\left(W_{\Psi}^{-1} \Phi W_{\Psi}^{-*}\right)
$$

where $\mathcal{F}: \mathcal{Q}_{m,+} \rightarrow \mathbb{R}$ is a suitable continuous function such that $\mathcal{F}(P) \geq 0$ and equality holds if and only if $P=I$. Here, we consider the following function parameterized by $\tau \in \mathbb{R} \backslash\{0,1\}$ :

$$
\mathcal{F}_{\tau}(P)=\operatorname{tr}\left[\frac{1}{\tau(\tau-1)} P^{\tau}-\frac{1}{\tau-1} P\right]+\frac{m}{\tau} .
$$

Substituting (6) in (5), we get the following divergence family:

$$
\begin{array}{r}
\mathcal{S}_{T}^{(\tau)}(\Phi \| \Psi):=\int \operatorname{tr}\left[\frac{1}{\tau(\tau-1)}\left(W_{\Psi}^{-1} \Phi W_{\Psi}^{-*}\right)^{\tau}\right. \\
\left.-\frac{1}{\tau-1} \Phi \Psi^{-1}\right]+\frac{m}{\tau} .
\end{array}
$$

Remark 2.1: The multivariate Alpha and Beta divergence family, [19], [18], are defined as follows, respectively:

$$
\begin{aligned}
\mathcal{S}_{A}^{(\alpha)}(\Phi \| \Psi) & :=\int \operatorname{tr}\left[\frac{1}{\alpha(\alpha-1)} \Phi^{\alpha} \Psi^{1-\alpha}-\frac{1}{\alpha-1} \Phi+\frac{1}{\alpha} \Psi\right] \\
\mathcal{S}_{B}^{(\beta)}(\Phi \| \Psi) & :=\int \operatorname{tr}\left[\frac{1}{\beta(\beta-1)} \Phi^{\beta}-\frac{1}{\beta-1} \Phi \Psi^{\beta-1}+\frac{1}{\beta} \Psi^{\beta}\right]
\end{aligned}
$$

where $\alpha, \beta \in \mathbb{R} \backslash\{0,1\}$. There exists a connection among the above divergences and $\mathcal{S}_{T}^{(\tau)}$ :

$$
\mathcal{S}_{T}^{(\tau)}(\Phi \| \Psi)=\mathcal{S}_{A}^{(\tau)}\left(W_{\Psi}^{-1} \Phi W_{\Psi}^{-*} \| I\right)=\mathcal{S}_{B}^{(\tau)}\left(W_{\Psi}^{-1} \Phi W_{\Psi}^{-*} \| I\right)
$$

Namely, $\mathcal{S}_{T}^{(\tau)}$ measures the dissimilarity between $E^{N}$ and $I$ through $\mathcal{S}_{A}^{(\tau)}$ or equivalently $\mathcal{S}_{B}^{(\tau)}$. 
Proposition 2.1: The following facts hold:

1) $\mathcal{S}_{T}^{(\tau)}$ can be extended by continuity for $\tau=0$ and $\tau=1$ :

$$
\begin{aligned}
& \lim _{\tau \rightarrow 0} \mathcal{S}_{T}^{(\tau)}(\Phi \| \Psi)=\mathcal{S}_{0}(\Phi \| \Psi) \\
& \lim _{\tau \rightarrow 1} \mathcal{S}_{T}^{(\tau)}(\Phi \| \Psi)=\mathcal{S}_{1}(\Phi \| \Psi)
\end{aligned}
$$

where

$$
\begin{aligned}
\mathcal{S}_{0}(\Phi \| \Psi):= & \int \operatorname{tr}\left[\log \Psi-\log \Phi+\Phi \Psi^{-1}\right]-m \\
\mathcal{S}_{1}(\Phi \| \Psi):= & \int \operatorname{tr}\left[W_{\Psi}^{-1} \Phi W_{\Psi}^{-*} \log \left(W_{\Psi}^{-1} \Phi W_{\Psi}^{-*}\right)\right. \\
& \left.-\Phi \Psi^{-1}\right]+m .
\end{aligned}
$$

2) $\mathcal{S}_{T}^{(\tau)}(\cdot \| \Psi)$ is strictly convex over $\mathcal{S}_{+}^{m}(\mathbb{T})$

3) $\mathcal{S}_{T}^{(\tau)}(\Phi \| \Psi) \geq 0$ and equality holds if and only if $\Phi=\Psi$.

Here, $\mathcal{S}_{0}$ is the multivariate Itakura-Saito distance, [4], between $\Phi$ and $\Psi$. Recalling that the multivariate Kullback-Leibler divergence extended to spectral densities with different zerothmoment is defined as, [18],

$$
\mathcal{S}_{\mathrm{KL}}\left(\Omega_{1} \| \Omega_{2}\right)=\int \operatorname{tr}\left[\Omega_{1}\left(\log \left(\Omega_{1}\right)-\log \left(\Omega_{2}\right)\right)-\Omega_{1}+\Omega_{2}\right]
$$

we conclude that $\mathcal{S}_{1}$ is the multivariate Kullback-Leibler divergence between $E^{N}$ and $I$.

Sketch of the Proof: Since $\Psi \in \mathcal{S}_{+}^{m}(\mathbb{T})$, the linear map $f_{\Psi}: \Phi \mapsto W_{\Psi}^{-1} \Phi W_{\Psi}^{-*}$ is bijective. Moreover, $\mathcal{S}_{T}^{(\tau)}(\Phi \| \Psi)=\mathcal{S}_{B}^{(\tau)}(\cdot \| I) \circ f_{\Psi}(\Phi)$. Thus, it is sufficient to apply Proposition 3.2 and Proposition 3.3 in [18] to get the statement.

$\mathcal{S}_{T}^{(\tau)}$ has been defined in (7) through the canonical left spectral factor $W_{\Psi}$ of $\Psi$, however it does not depend on this particular choice.

Proposition 2.2: Let $\bar{W}_{\Psi}$ be any left square spectral factor of $\Psi$. Define

$$
\begin{array}{r}
\overline{\mathcal{S}}_{T}^{(\tau)}(\Phi \| \Psi)=\int \operatorname{tr}\left[\frac{1}{\tau(\tau-1)}\left(\bar{W}_{\Psi}^{-1} \Phi \bar{W}_{\Psi}^{-*}\right)^{\tau}\right. \\
\left.-\frac{1}{\tau-1} \Phi \Psi^{-1}\right]+\frac{m}{\tau}
\end{array}
$$

where $\tau \in \mathbb{R} \backslash\{0,1\}$. Then,

$$
\mathcal{S}_{T}^{(\tau)}(\Phi \| \Psi)=\overline{\mathcal{S}}_{T}^{(\tau)}(\Phi \| \Psi)
$$

and this equality also holds for $\tau \rightarrow 0$ and $\tau \rightarrow 1$. 
Proof: The spectral factor $\bar{W}_{\Psi}$ can be obtained through the canonical one as follows

$$
\bar{W}_{\Psi}=W_{\Psi} U^{*}
$$

where $U$ is an $m \times m$ all pass function, i.e. $U U^{*}=I$ on $\mathbb{T}$. Let $\bar{W}_{\Psi}^{-1} \Phi \bar{W}_{\Psi}^{-*}=V D V^{*}$ be a pointwise SVD of $\bar{W}_{\Psi}^{-1} \Phi \bar{W}_{\Psi}^{-*}$, therefore $D$ is diagonal and $V V^{*}=I$ on $\mathbb{T}$. Hence,

$$
\begin{aligned}
\bar{W}_{\Psi}^{-1} \Phi \bar{W}_{\Psi}^{-*} & =\left(W_{\Psi} U^{*}\right)^{-1} \Phi\left(W_{\Psi} U^{*}\right)^{-*} \\
& =U W_{\Psi}^{-1} \Phi W_{\Psi}^{-*} U^{*}=U V D V^{*} U^{*}
\end{aligned}
$$

and $U V$ is an $m \times m$ all pass function, in fact

$$
(U V)(U V)^{*}=U V V^{*} U^{*}=U U^{*}=I .
$$

Thus, $V D V^{*}$ and $(U V) D(U V)^{*}$ are two pointwise SVD of $\bar{W}_{\Psi}^{-1} \Phi \bar{W}_{\Psi}^{-*}$. In order to show that 13. holds for $\tau \in \mathbb{R} \backslash\{0,1\}$, it is sufficient to show that $\operatorname{tr}\left[\left(W_{\Psi}^{-1} \Phi W_{\Psi}^{-*}\right)^{\tau}\right]=\operatorname{tr}\left[\left(\bar{W}_{\Psi}^{-1} \Phi \bar{W}_{\Psi}^{-*}\right)^{\tau}\right]$ :

$$
\begin{aligned}
& \operatorname{tr}\left[\left(\bar{W}_{\Psi}^{-1} \Psi \bar{W}_{\Psi}^{-*}\right)^{\tau}\right]=\operatorname{tr}\left[\left(U V D V^{*} U^{*}\right)^{\tau}\right] \\
& =\operatorname{tr}\left[U V D^{\tau} V^{*} U^{*}\right]=\operatorname{tr}\left[V D^{\tau} V^{*}\right] \\
& \quad=\operatorname{tr}\left[\left(V D V^{*}\right)^{\tau}\right]=\operatorname{tr}\left[\left(W_{\Psi}^{-1} \Psi W_{\Psi}^{-*}\right)^{\tau}\right]
\end{aligned}
$$

accordingly $\mathcal{S}_{T}^{(\tau)}(\Phi \| \Psi)=\overline{\mathcal{S}}_{T}^{(\tau)}(\Phi \| \Psi)$. For the limit cases, we have

$$
\begin{gathered}
\lim _{\tau \rightarrow 0} \mathcal{S}_{\tau}(\Phi \| \Psi)=\int \operatorname{tr}\left[\log \Psi-\log \Phi+\Phi \Psi^{-1}\right]-m \\
\lim _{\tau \rightarrow 1} \mathcal{S}_{\tau}(\Phi \| \Psi)=\int \operatorname{tr}\left[\bar{W}_{\Psi}^{-1} \Psi \bar{W}_{\Psi}^{-*} \log \left(\bar{W}_{\Psi}^{-1} \Psi \bar{W}_{\Psi}^{-*}\right)\right. \\
\left.-\Phi \Psi^{-1}\right]+m
\end{gathered}
$$

Therefore, (13) holds for $\tau \rightarrow 0$. For $\tau \rightarrow 1$ we have

$$
\begin{aligned}
\operatorname{tr} & {\left[\bar{W}_{\Psi}^{-1} \Psi \bar{W}_{\Psi}^{-*} \log \left(\bar{W}_{\Psi}^{-1} \Psi \bar{W}_{\Psi}^{-*}\right)\right] } \\
& =\operatorname{tr}\left[U V D V^{*} U^{*} U V \log (D) V^{*} U^{*}\right] \\
& =\operatorname{tr}\left[V D V^{*} V \log (D) V^{*}\right] \\
& =\operatorname{tr}\left[W_{\Psi}^{-1} \Psi W_{\Psi}^{-*} \log \left(W_{\Psi}^{-1} \Psi W_{\Psi}^{-*}\right)\right]
\end{aligned}
$$

therefore (13) holds for $\tau \rightarrow 1$. 
It is worth noting that $\mathcal{S}_{T}^{(\tau)}$ remains invariant under congruence, that is

$$
\mathcal{S}_{T}^{(\tau)}(\Phi \| \Psi)=\mathcal{S}_{T}^{(\tau)}\left(T^{*} \Phi T \| T^{*} \Psi T\right)
$$

for any $\mathbb{C}^{m \times m}$-valued function $T(z)$ invertible on $\mathbb{T}$. This is a natural property to demand since it implies that the divergence between spectral densities does not change under coordinate transformation. In particular, $\mathcal{S}_{T}^{(\tau)}$ is invariant to scaling: such a property seems essential, for instance, for speech and image systems, due to an apparent agreement with subjective qualities of sound and images. This invariance property, however, does not hold for $\mathcal{S}_{A}^{(\alpha)}$ and $\mathcal{S}_{B}^{(\beta)}$. Finally, it is worth noting that $\mathcal{S}_{T}^{(\tau)}$ is not even known in the scalar case (i.e. $m=1$ ).

\section{THREE-LIKE SPECTRAL ESTIMATION}

Consider the stochastic process $y$ of Section II with unknown spectral density $\Phi\left(\mathrm{e}^{j \vartheta}\right) \in \mathcal{S}_{+}^{m}(\mathbb{T})$. Assume that, the given a priori spectral density $\Psi \in \mathcal{S}_{+}^{m}(\mathbb{T})$ has bounded McMillan degree. Then, a finite length sequence $\mathbf{y}_{1} \ldots \mathbf{y}_{N}$ extracted from a realization of $y$ is available. We want to find an estimate of $\Phi$ by exploiting $\Psi$ and $\mathbf{y}_{1} \ldots \mathbf{y}_{N}$. According to the THREE-like approach, we design a rational filter

$$
G^{\prime}(z)=(z I-A)^{-1} B
$$

where $A \in \mathbb{R}^{n \times n}$ is a stability matrix, $B \in \mathbb{R}^{n \times m}$ is full rank with $n>m$ and $(A, B)$ is a reachable pair. We compute an estimate $\hat{\Sigma} \succ 0$, based on the data $\mathbf{y}_{1} \ldots \mathbf{y}_{N}$, of the steady state covariance $\Sigma=\Sigma^{T} \succ 0$ of the state $x_{k}$ of the filter

$$
x_{k+1}=A x_{k}+B y_{k} .
$$

Then, an estimate of $\Phi$ is given by solving the following spectrum approximation problem.

Problem 1: Given $\Psi \in \mathcal{S}_{+}^{m}(\mathbb{T}), G(z)=\hat{\Sigma}^{-\frac{1}{2}}(z I-A)^{-1} B$, and $\nu \in \mathbb{N}_{+}:=\mathbb{N} \backslash\{0\}$,

$$
\begin{aligned}
& \operatorname{minimize} \mathcal{S}_{T}^{\left(1-\nu^{-1}\right)}(\Phi \| \Psi) \text { over the set } \\
& \qquad \mathcal{J}=\left\{\Phi \in \mathcal{S}_{+}^{m}(\mathbb{T}) \mid \int G \Phi G^{*}=I\right\} .
\end{aligned}
$$

The constraint in $(23)$ is equivalent to

$$
\int G^{\prime} \Phi\left(G^{\prime}\right)^{*}=\hat{\Sigma}
$$

Accordingly, the optimal solution to Problem 1 (if it does exist) matches the data encoded by $\hat{\Sigma}$ and is such that the one-step-ahead predictor based on $\Psi$ is as close as possible to be the 
optimal one. Note that, the set $\mathcal{J}$ depends on the estimate $\hat{\Sigma}$. In this section we assume that $\hat{\Sigma}$ is chosen in such a way that $\mathcal{J}$ is non-empty, that is Problem 1 is feasible. In Section IV, we will show how to compute such a $\hat{\Sigma}$.

In [4], it was already shown that Problem 1 admits a unique solution for $\nu=1$. Thus, we deal with the case $\nu>1$. Since Problem 1 is a constrained convex optimization problem, we consider the corresponding Lagrange functional

$$
\begin{aligned}
L_{\nu}(\Phi, \Lambda) & \\
= & \mathcal{S}_{T}^{\left(1-\nu^{-1}\right)}(\Phi \| \Psi)-m \frac{\nu}{\nu-1}+\left\langle\int G \Phi G^{*}-I, \Lambda\right\rangle \\
= & \int \operatorname{tr}\left[\frac{\nu^{2}}{1-\nu}\left(W_{\Psi}^{-1} \Phi W_{\Psi}^{-*}\right)^{\frac{\nu-1}{\nu}}+\nu\left(W_{\Psi}^{-1} \Phi W_{\Psi}^{-*}\right)\right. \\
\quad & \left.+G^{*} \Lambda G \Phi\right]-\operatorname{tr}[\Lambda]
\end{aligned}
$$

where we exploited the fact that the term $m \frac{\nu}{\nu-1}$ plays no role in the optimization problem. Note that, the domain of $L_{\nu}(\cdot, \Lambda)$ is $\mathcal{S}_{+}^{m}(\mathbb{T})$ and $\Lambda \in \mathcal{Q}_{n}$ is the Lagrange multiplier associated to the constraint in 23 . Consider the vector space

$$
\mathcal{Q}_{n}^{G}:=\left\{\int G \Phi G^{*} \text { s.t. } \Phi \in \mathcal{V}\left(\mathcal{S}_{+}^{m}(\mathbb{T})\right)\right\}
$$

where $\mathcal{V}\left(\mathcal{S}_{+}^{m}(\mathbb{T})\right)$ denotes the vector space generated by $\mathcal{S}_{+}^{m}(\mathbb{T})$. In [17], it was shown that $\Lambda$ can be uniquely decomposed as $\Lambda_{G}+\Lambda_{\perp}$ where $\Lambda_{G} \in \mathcal{Q}_{n}^{G}$ and $\Lambda_{\perp} \in\left(\mathcal{Q}_{n}^{G}\right)^{\perp}$. Moreover, $G^{*}\left(\mathrm{e}^{i \vartheta}\right) \Lambda_{\perp} G\left(\mathrm{e}^{i \vartheta}\right) \equiv 0$ and $\operatorname{tr}\left[\Lambda_{\perp}\right]=0$. Accordingly, $\Lambda_{\perp}$ does not affect the Lagrange functional and we can restrict $\Lambda \in \mathcal{Q}_{n}^{G}$. Since $L_{\nu}(\cdot, \Lambda)$ is strictly convex over $\mathcal{S}_{+}^{m}(\mathbb{T})$, it is sufficient to show the existence of a stationary point for $L_{\nu}(\cdot, \Lambda)$ in order to prove the existence of a (unique) solution to the unconstrained minimization problem $\Phi_{\nu}(\Lambda):=\arg \min _{\Phi}\left\{L_{\nu}(\Phi, \Lambda) \mid \Phi \in \mathcal{S}_{+}^{m}(\mathbb{T})\right\}$. The first variation of $L_{\nu}(\cdot, \Lambda)$ in each direction $\delta \Phi \in L_{\infty}^{m \times m}(\mathbb{T})$ is:

$$
\begin{aligned}
& \delta L_{\nu}(\Phi, \Lambda ; \delta \Phi)=\int \operatorname{tr}\left[-\nu\left(W_{\Psi}^{-1} \Phi W_{\Psi}^{-*}\right)^{-\frac{1}{\nu}} W_{\Psi}^{-1} \delta \Phi W_{\Psi}^{-*}\right. \\
& \left.\quad+\nu W_{\Psi}^{-1} \delta \Phi W_{\Psi}^{-*}+G^{*} \Lambda G \delta \Phi\right] \\
& =\int \operatorname{tr}\left[\left(-\nu W_{\Psi}^{-*}\left(W_{\Psi}^{-1} \Phi W_{\Psi}^{-*}\right)^{-\frac{1}{\nu}} W_{\Psi}^{-1}\right.\right. \\
& \left.\left.\quad+\nu W_{\Psi}^{-*} W_{\Psi}^{-1}+G^{*} \Lambda G\right) \delta \Phi\right]
\end{aligned}
$$


where we used the expression of the first variation of the exponentiation of $X \in \mathcal{Q}_{n,+}$ to $c \in \mathbb{R}$ given in [18]:

$$
\delta\left(\operatorname{tr}\left[X^{c}\right] ; \delta X\right)=c \operatorname{tr}\left[X^{c-1}\right], \quad \delta X \in \mathcal{Q}_{n}
$$

Note that, $-\nu W_{\Psi}^{-*}\left(W_{\Psi}^{-1} \Phi W_{\Psi}^{-*}\right)^{-\frac{1}{\nu}} W_{\Psi}^{-1}+\nu W_{\Psi}^{-*} W_{\Psi}^{-1}+G^{*} \Lambda G \in L_{\infty}^{m \times m}(\mathbb{T})$. Thus, 27, is zero $\forall \delta \Phi \in L_{\infty}^{m \times m}(\mathbb{T})$ if and only if

$$
\left(W_{\Psi}^{-1} \Phi W_{\Psi}^{-*}\right)^{-\frac{1}{\nu}}=I+\frac{1}{\nu} W_{\Psi}^{*} G^{*} \Lambda G W_{\Psi}
$$

Since $\left(W_{\Psi}^{-*} \Phi W_{\Psi}^{-*}\right)^{-\frac{1}{\nu}} \in \mathcal{S}_{+}^{m}(\mathbb{T})$, the set of the admissible Lagrange multipliers is

$$
\mathcal{L}_{+}:=\left\{\Lambda \in \mathcal{Q}_{n}^{G} \mid I+\frac{1}{\nu} W_{\Psi}^{*} G^{*} \Lambda G W_{\Psi} \succ 0 \text { on } \mathbb{T}\right\} .
$$

Therefore, if $\Lambda \in \mathcal{L}_{+}$then the unique stationary point for $L_{\nu}(\cdot, \Lambda)$ is

$$
\Phi_{\nu}(\Lambda):=W_{\Psi}\left(I+\frac{1}{\nu} W_{\Psi}^{*} G^{*} \Lambda G W_{\Psi}\right)^{-\nu} W_{\Psi}^{*}
$$

which coincides with the unique minimum point for $L_{\nu}(\cdot, \Lambda)$.

Proposition 3.1: If $\Phi_{\nu}$ is a minimizer of Problem 1, then it has bounded McMillan degree which is less than or equal to $\nu(\operatorname{deg}[\Psi]+2 n)$. Moreover the following facts hold:

1) Among all the spectral densities $\Phi_{\nu}$ with $\nu \in \mathbb{N}_{+}$, the spectral density with the smallest upper bound on the McMillan degree corresponds to $\mathcal{S}_{0}$ defined in (10)

2) As $\nu \rightarrow+\infty, \Phi_{\nu}$ converges to the optimal form corresponding to $\mathcal{S}_{1}$ defined in (10).

Proof: In [4], it was shown that (31) holds for $\nu=1$ and $\operatorname{deg}\left[\Phi_{1}\right] \leq \operatorname{deg}[\Psi]+2 n$. For the case $\nu>1, \Phi_{\nu}(\Lambda)=L_{\Lambda} Q_{\Lambda}^{\nu-2} L_{\Lambda}^{T}$ where $Q_{\Lambda}:=\left(I+\frac{1}{\nu} W_{\Psi}^{*} G^{*} \Lambda G W_{\Psi}\right)^{-1}, L_{\Lambda}:=\left(W_{\Psi}^{-1}+W_{\Psi}^{*} G^{*} \Lambda G\right)^{-1}$, $G$ has bounded McMillan degree by assumption, and $W_{\Psi}$ has bounded degree because is the canonical left spectral factor of a spectral density with bounded degree. Since $\operatorname{deg}\left[Q_{\Lambda}\right]$ and $\operatorname{deg}\left[L_{\Lambda}\right]$ are less than or equal to $\operatorname{deg}[\Psi]+2 n$, we conclude that $\Phi_{\nu}(\Lambda)$ has bounded McMillan degree which is less than or equal to $\nu(\operatorname{deg}[\Psi]+2 n)$.

Point 1. Since $\nu(\operatorname{deg}[\Psi]+2 n)$ is an increasing function in $\nu \in \mathbb{N}_{+}$, its minimum is achieved with $\nu=1$, i.e. with $\mathcal{S}_{T}^{(0)}=\mathcal{S}_{0}$.

Point 2. It is not difficult to show that the optimal form obtained by using $\mathcal{S}_{1}$ in Problem 1 is $\Phi_{\infty}(\Lambda):=W_{\Psi} \mathrm{e}^{-W_{\Psi}^{*} G^{*} \Lambda G W_{\Psi}} W_{\Psi}^{*}$. By using the limit, see [18, Proposition 4.1]:

$$
\lim _{\nu \rightarrow \infty}\left(I+\frac{1}{\nu} X\right)^{-\nu}=\mathrm{e}^{-X}, \quad X \in \mathcal{Q}_{n}
$$


we get

$$
\lim _{\nu \rightarrow \infty} \Phi_{\nu}(\Lambda)=W_{\Psi} \mathrm{e}^{-W_{\Psi}^{*} G^{*} \Lambda G W_{\Psi}} W_{\Psi}^{*}=\Phi_{\infty}(\Lambda)
$$

Remark 3.1: In [18], the multivariate Beta divergence family $\mathcal{S}_{B}^{\left(1-\nu^{-1}\right)}$ with $\nu \in \mathbb{N}_{+}$has been considered. The optimal form of the spectrum approximation problem is

$$
\Phi_{B, \nu}(\Lambda):=\left(\Psi^{-\frac{1}{\nu}}+\frac{1}{\nu} G^{*} \Lambda G\right)^{-\nu}
$$

and the assumption that $\Psi$ has bounded McMillan degree is not sufficient to guarantee that $\Psi^{\frac{1}{\nu}}$, and also $\Phi_{B, \nu}$, has bounded degree. In [19], the scalar Alpha divergence family $\mathcal{S}_{A}^{\left(1-\nu^{-1}\right)}$ with $\nu \in \mathbb{N}_{+}$has been considered. The corresponding optimal form is

$$
\Phi_{A, \nu}(\Lambda):=\frac{\Psi}{\left(I+\frac{1}{\nu} G^{*} \Lambda G\right)^{\nu}}
$$

and $\Psi$ with bounded McMIllan degree implies that $\Phi_{A, \nu}$ has bounded degree. However, such a result cannot be extended to the multichannel case, i.e. $m>1$. In view of Proposition 3.1, $\mathcal{S}_{T}^{\left(1-\nu^{-1}\right)}$ with $\nu \in \mathbb{N}_{+}$is the unique divergence family always leading to a bounded degree optimal form in the multichannel case once $\Psi$ has bounded degree.

Since $\Phi_{\nu}$ is the unique minimum point for $L_{\nu}(\cdot, \Lambda)$ over $\mathcal{S}_{+}^{m}(\mathbb{T})$, if we produce $\Lambda^{\circ} \in \mathcal{L}_{+}$such that $\int G \Phi_{\nu}\left(\Lambda^{\circ}\right) G^{*}=I$, then $\Phi_{\nu}\left(\Lambda^{\circ}\right)$ is the unique solution to Problem 1 . To this end, consider the dual functional defined over $\mathcal{L}_{+}$:

$$
J_{\nu}(\Lambda)=\frac{\nu}{1-\nu} \int \operatorname{tr}\left[\left(I+\frac{1}{\nu} W_{\Psi}^{*} G^{*} \Lambda G W_{\Psi}\right)^{1-\nu}\right]-\operatorname{tr}[\Lambda] .
$$

Theorem 3.1: If $\mathcal{J}$ is a non-empty set, then the dual problem $\max \left\{J_{\nu}(\Lambda) \mid \Lambda \in \mathcal{L}_{+}\right\}$with $\nu>1$ admits a unique solution. Such a solution, say $\Lambda^{\circ}$, satisfies

$$
\int G \Phi_{\nu}\left(\Lambda^{\circ}\right) G^{*}=I
$$


Proof: Recall that $Q_{\Lambda}=\left(I+\frac{1}{\nu} W_{\Psi}^{*} G^{*} \Lambda G W_{\Psi}\right)^{-1}$. By similar argumentations used in [18, Theorem 5.1], it is possible to show that $J_{\nu} \in \mathcal{C}^{2}\left(\mathcal{L}_{+}\right)$, and

$$
\begin{aligned}
& \delta J_{\nu}(\Lambda ; \delta \Lambda) \\
& =\operatorname{tr} \int\left[Q_{\Lambda}^{\nu} W_{\Psi}^{*} G^{*} \delta \Lambda G W_{\Psi}\right]-\operatorname{tr}[\delta \Lambda] \\
& \delta^{2} J_{\nu}(\Lambda ; \delta \Lambda) \\
& =-\frac{1}{\nu} \sum_{l=1}^{\nu} \int \operatorname{tr}\left[Q_{\Lambda}^{l} W_{\Psi}^{*} G^{*} \delta \Lambda G W_{\Psi} Q_{\Lambda}^{\nu+1-l}\right. \\
& \left.\quad \times W_{\Psi}^{*} G^{*} \delta \Lambda G W_{\Psi}\right]
\end{aligned}
$$

where we used the fact that the first variation of $Q_{\Lambda}$ in direction $\delta \Lambda \in \mathcal{Q}_{n}$ is

$$
Q_{\Lambda ; \delta \Lambda}=-\frac{1}{\nu} Q_{\Lambda} W_{\Psi}^{*} G^{*} \delta \Lambda G W_{\Psi} Q_{\Lambda}
$$

and the first variation of the map $\mathcal{I}: A \mapsto A^{\nu}$ in direction $\delta A \in \mathcal{Q}_{n}$ is:

$$
\delta(\mathcal{I}(A) ; \delta A)=\sum_{l=1}^{\nu} A^{l-1} \delta A A^{\nu-l} .
$$

Since $\nu>1$ and the trace of the integrands in (39) is nonnegative, we have $\delta^{2} J_{\nu} \leq 0$. If $\delta^{2} J_{\nu}=0$, then $G^{*} \delta \Lambda G \equiv 0$, namely $\delta \Lambda \in\left(\mathcal{Q}_{n}^{G}\right)^{\perp}$, see [17]. Since $\delta \Lambda \in \mathcal{Q}_{n}^{G}$, we conclude that $\delta \Lambda=0$. This means that $\delta^{2} J_{\nu}$ is negative definite. Thus, $J_{\nu}$ is strictly concave on $\mathcal{L}_{+}$and the dual problem admits at most one solution $\Lambda^{\circ}$ which must annihilate $(38)$ for each $\delta \Lambda$. This implies that $\Lambda^{\circ}$ satisfies (37).

It remains to be shown that $J_{\nu}$ takes a maximum value on $\mathcal{L}_{+}$. Note that $J_{\nu}(0)=m \frac{\nu}{1-\nu}$, accordingly we can restrict the search of a maximum point to the nonempty set $\mathcal{K}:=\{\Lambda \in$ $\mathcal{L}_{+}$s.t. $\left.J_{\nu}(\Lambda) \geq J_{\nu}(0)\right\}$. By similar argumentations used in [18, Theorem 5.2], it is possible to show that

$$
\begin{aligned}
& \lim _{\Lambda \rightarrow \partial \mathcal{L}_{+}} J_{\nu}(\Lambda)=-\infty \\
& \lim _{\|\Lambda\| \rightarrow \infty} J_{\nu}(\Lambda)=-\infty
\end{aligned}
$$

that is $\mathcal{K}$ is compact. Finally, since $J_{\nu} \in \mathcal{C}^{2}\left(\mathcal{L}_{+}\right)$, the existence of the solution follows from the Weierstrass' Theorem.

The optimal solution $\Lambda^{\circ}$ can be efficiently computed by using the matricial Newton algorithm with backtracking presented in [17]. Here, the Newton step $\Delta_{\Lambda_{i}}$ at the $i$-th iteration with starting 
point $\Lambda_{i}$ is given by solving the linear equation

$$
\begin{array}{r}
\frac{1}{\nu} \sum_{l=1}^{\nu} \int G W_{\Psi} Q_{\Lambda_{i}}^{l} W_{\Psi}^{*} G^{*} \Delta_{\Lambda_{i}} G W_{\Psi} Q_{\Lambda_{i}}^{\nu+1-l} W_{\Psi}^{*} G^{*} \\
=\int G W_{\Psi} Q_{\Lambda_{i}}^{\nu} W_{\Psi}^{*} G^{*}-I .
\end{array}
$$

Concerning its computation, in [17] a sensible and efficient method based on spectral factorization techniques has been presented. Finally, it is possible to prove that this algorithm globally converges, in particular the rate of convergence is quadratic during the last stage.

\section{State Covariance Estimation}

In this section we face the problem of computing $\hat{\Sigma}$ in such a way that the set $\mathcal{J}$ is non-empty. In [6], (see also [8], [5]) it was shown that $\mathcal{J}$ is non-empty if and only if $\hat{\Sigma}$ is positive definite and it belongs to the kernel of the linear operator

$$
\begin{aligned}
V: \mathcal{Q}_{n} & \rightarrow \mathcal{Q}_{n} \\
Q & \mapsto \Pi_{B}^{\perp}\left(Q-A Q A^{T}\right) \Pi_{B}^{\perp},
\end{aligned}
$$

where $\Pi_{B}^{\perp}=I-B\left(B^{T} B\right)^{-1} B^{T}$. Thus, we can feed the bank of filters $G^{\prime}(z)$ with the finite length sequence $\mathbf{y}_{1} \ldots \mathbf{y}_{N}$, obtaining the output data $\mathbf{x}_{1} \ldots \mathbf{x}_{N}$. Then an estimate of $\Sigma$ is given by the sample covariance $\hat{\Sigma}_{C}:=\sum_{k=1}^{N} \mathbf{x}_{k} \mathbf{x}_{k}^{T}$ which is normally positive definite, but it may happen $V\left(\hat{\Sigma}_{C}\right) \neq 0$ especially when $N$ is not large. Following the same approach presented in [6], [18], an estimate of $\Sigma$ leading to a non-empty set $\mathcal{J}$ is given by finding a new positive definite estimate $\hat{\Sigma}$ such that $V(\hat{\Sigma})=0$ and "close" to the estimate $\hat{\Sigma}_{C}$.

For measuring the closeness between $\hat{\Sigma}$ and $\hat{\Sigma}_{C}$, we consider the following divergence index between $P, Q \in \mathcal{Q}_{n,+}$ with $\tau \in \mathbb{R} \backslash\{0,1\}$ :

$$
\mathcal{D}_{T}^{(\tau)}(P \| Q):=\operatorname{tr}\left[\frac{1}{\tau(\tau-1)}\left(L_{Q}^{-1} P L_{Q}^{-T}\right)^{\tau}-\frac{1}{\tau-1}\left(P Q^{-1}\right)\right]+\frac{m}{\tau} .
$$

Here, $L_{Q}$ is the Cholesky decomposition of $Q$, i.e. $Q=L_{Q} L_{Q}^{T}$. Note that $\mathcal{D}_{T}^{(\tau)}$ is a special case of $\mathcal{S}_{T}^{(\tau)}$ : it is sufficient to pick $\Phi\left(\mathrm{e}^{i \vartheta}\right)=P$ and $\Psi\left(\mathrm{e}^{i \vartheta}\right)=Q$ in order to obtain 45 . Accordingly, $\mathcal{D}_{T}^{(\tau)}$ is strictly convex with respect to the first argument and it can be extended by continuity for $\tau=0$ and $\tau=1$ :

$$
\lim _{\tau \rightarrow 0} \mathcal{D}_{T}^{(\tau)}(P \| Q)=\mathcal{D}_{0}(P \| Q), \lim _{\tau \rightarrow 1} \mathcal{D}_{T}^{(\tau)}(P \| Q)=\mathcal{D}_{1}(P \| Q)
$$


where

$$
\begin{aligned}
\mathcal{D}_{0}(P \| Q):= & {\left[\operatorname{tr} \int \log Q-\log P+P Q^{-1}\right]-m } \\
\mathcal{D}_{1}(P \| Q):= & {\left[\operatorname{tr} \int\left(L_{Q}^{-1} P L_{Q}^{-T}\right) \log \left(L_{Q}^{-1} P L_{Q}^{-T}\right)\right.} \\
& \left.-L_{Q}^{-1} P L_{Q}^{-T}\right]+m .
\end{aligned}
$$

Here, $\mathcal{D}_{0}$ is the Burg matrix divergence and $\mathcal{D}_{1}$ is the Umegaki-von Neumann's relative entropy (extended to non-equal trace matrices) between $L_{Q}^{-1} P L_{Q}^{-T}$ and $I$.

We are now ready to introduce the optimization problem for finding $\hat{\Sigma}$.

Problem 2: Given $\hat{\Sigma}_{C} \succ 0$ and $\nu \in \mathbb{N}_{+}$, solve

$$
\begin{aligned}
& \operatorname{minimize} \mathcal{D}_{T}^{\left(1-\nu^{-1}\right)}\left(\hat{\Sigma} \| \hat{\Sigma}_{C}\right) \text { over the set } \\
& \left.\qquad \hat{\Sigma} \in \mathcal{Q}_{n,+} \mid V(\hat{\Sigma})=0\right\} .
\end{aligned}
$$

Theorem 4.1: Problem 2 admits a unique solution.

Proof: Since $\mathcal{D}_{T}^{\left(1-\nu^{-1}\right)}\left(\cdot \| \hat{\Sigma}_{C}\right)$ is strictly convex on $\mathcal{Q}_{n,+}$, Problem 2 admits at most one solution. Then, the existence of such a solution can be proved by duality theory as in [18, Section VIII].

Also in this case, a globally convergent matricial Newton algorithm for computing $\hat{\Sigma}$ may be used, see [6]. Finally, it is worth noting that it is also possible to estimate $\Sigma$ through the estimates of the covariance lags of the input process $y$, [20], [7].

\section{Simulation Study}

In this section we want to test the features of the family of estimators $\Phi_{\nu}$ through a simulation study. Assume that a finite length sequence $\mathbf{y}_{1} \ldots \mathbf{y}_{N}$ is extracted from a realization of a bivariate process $y$ with spectral density $\Phi \in \mathcal{S}_{+}^{m}(\mathbb{T})$. We want to compute the estimates $\Phi_{\nu}$ with $\nu=1$ and $\nu=2$ of $\Phi$. In view of the results of Section III and Section IV, we consider the following identification procedure:

- Choose a low order a priori spectral density $\Psi$ with bounded McMillan degree;

- Choose $G^{\prime}(z)$ as in Section III, and $\nu \in \mathbb{N}_{+}$; 
- Feed the bank of filters $G^{\prime}$ with the sequence $\mathbf{y}_{1} \ldots \mathbf{y}_{N}$, collect the output data $\mathbf{x}_{1} \ldots \mathbf{x}_{N}$ and compute $\hat{\Sigma}_{C}=\frac{1}{N} \sum_{k=1}^{N} \mathbf{x}_{k} \mathbf{x}_{k}^{T}$;

- Compute $\hat{\Sigma}_{\nu}$ by solving Problem 2 ,

- Compute $\Phi_{\nu}$ by solving Problem 1 with the chosen $\Psi$ and $G(z)=\hat{\Sigma}_{\nu}^{-\frac{1}{2}} G^{\prime}(z)$.

In the above procedure we have two degrees of freedom: the way for choosing $\Psi$ and the structure of $G^{\prime}(z)$.

The a priori spectral density $\Psi$ may be derived from given laws (e.g. physical laws if $y$ represents a physical phenomenon) or from the data by applying a (simple) identification method. In both cases $\Psi$ represents a coarse, low order, estimate of $\Phi$. In our case, $\Psi$ is an $\operatorname{ARMA}(1,1)$ which is computed from $\mathbf{y}_{1} \ldots \mathbf{y}_{N}$ by applying the MATLAB's PEM identification method.

Concerning the design of $G^{\prime}(z)=(z I-A)^{-1} B$, a higher resolution can be attained by selecting poles in the proximity of the unit circle, with arguments in the range of frequency of interest, [3]. Here, we choose

$$
A=\left[\begin{array}{llll}
0 & I & 0 & 0 \\
0 & 0 & I & 0 \\
0 & 0 & 0 & I \\
0 & 0 & 0 & 0
\end{array}\right] \quad B=\left[\begin{array}{l}
0 \\
0 \\
0 \\
I
\end{array}\right]
$$

which implies that $\Sigma$ is a block Toeplitz matrix whose block diagonals contain the lags $\mathbb{E}\left[y_{k} y_{k+j}^{T}\right]$ $j=0,1,2,3$ of the covariance function of $y$. Accordingly, Problem 1 becomes a covariance extension problem.

Finally, the sequence $\mathbf{y}_{1} \ldots \mathbf{y}_{N}$ is generated by feeding a bivariate WGN with zero mean and variance $I$ to a (stable) square shaping filter of order 40. The latter is constructed with random coefficients.

We consider four different lengths for the sequence: $N=100, N=500, N=1000$ and $N=2000$, i.e. we start from short observation records up to long observation records. For each $N$, in order to obtain a comparison reasonably independent of the specific data set, we perform 50 independent runs where the sequence $\mathbf{y}_{1} \ldots \mathbf{y}_{N}$ changes in each run. In this way, we obtain 50 different estimates $\Phi_{\nu, k}^{N} k=1 \ldots 50$. We define then

$$
\operatorname{err}_{\nu}^{N}(k):=\int \frac{\left\|\Phi_{\nu, k}^{N}-\Phi\right\|}{\|\Phi\|}
$$


where $\|\cdot\|$ denotes the spectral norm. This is understood as the relative estimation error of $\Phi_{\nu, k}^{N}$ averaged over the unit circle. In Figure 1 boxplots of the averaged error achieved by the

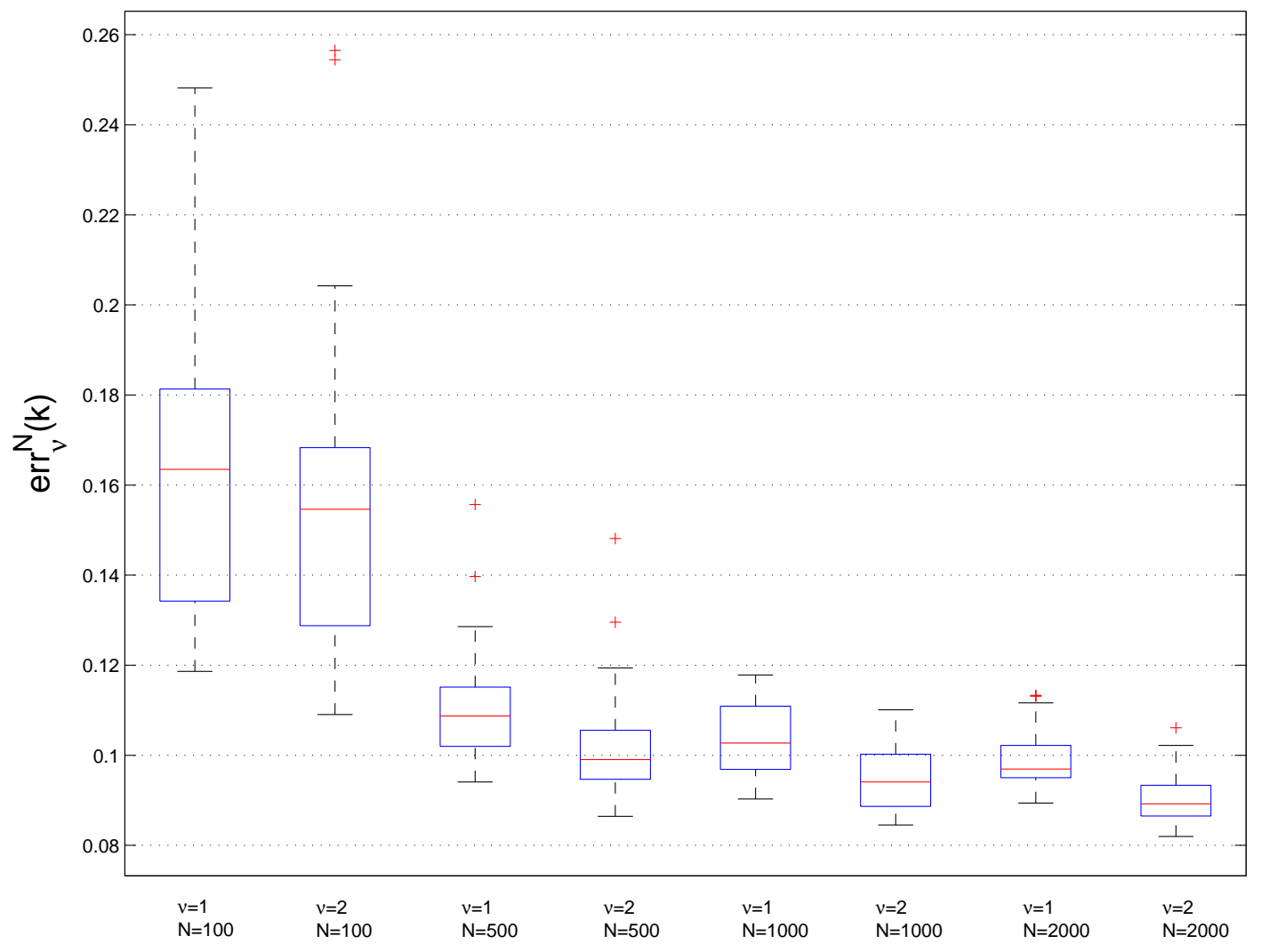

Fig. 1. Boxplots of the relative errors averaged over the unit circle for different values of $N$, and $\nu=1$ and $\nu=2$.

estimates with $\nu=1$ and $\nu=2$ for different values of $N$ is depicted. Clearly, the larger $N$ is the better estimates are. Moreover, the estimator with $\nu=2$ always outperforms the one with $\nu=1$. On the other hand, the McMillan degree of $\Phi_{\nu}$ increases by increasing $\nu$ : the spectral factor (i.e. the model) with $\nu=1$ has degree 9 , whereas with $\nu=2$ has degree 18 . The spectral density of the normalized innovation process corresponding to $\Phi_{\nu, k}^{N}$ is $E_{\nu, k}^{N}:=W_{\Psi}^{-1} \Phi_{\nu, k}^{N} W_{\Psi}^{-*}$. We define

$$
E_{\nu}^{N}=\frac{1}{50} \sum_{k=1}^{50} E_{\nu, k}^{N}
$$


which is the spectral density of the normalized innovation process averaged over 50 runs and $N$ is fixed. In Figure 2, $E_{\nu}^{100}$ with $\nu=1$ and $\nu=2$ is depicted. We observe that $E_{2}^{100}$ is more
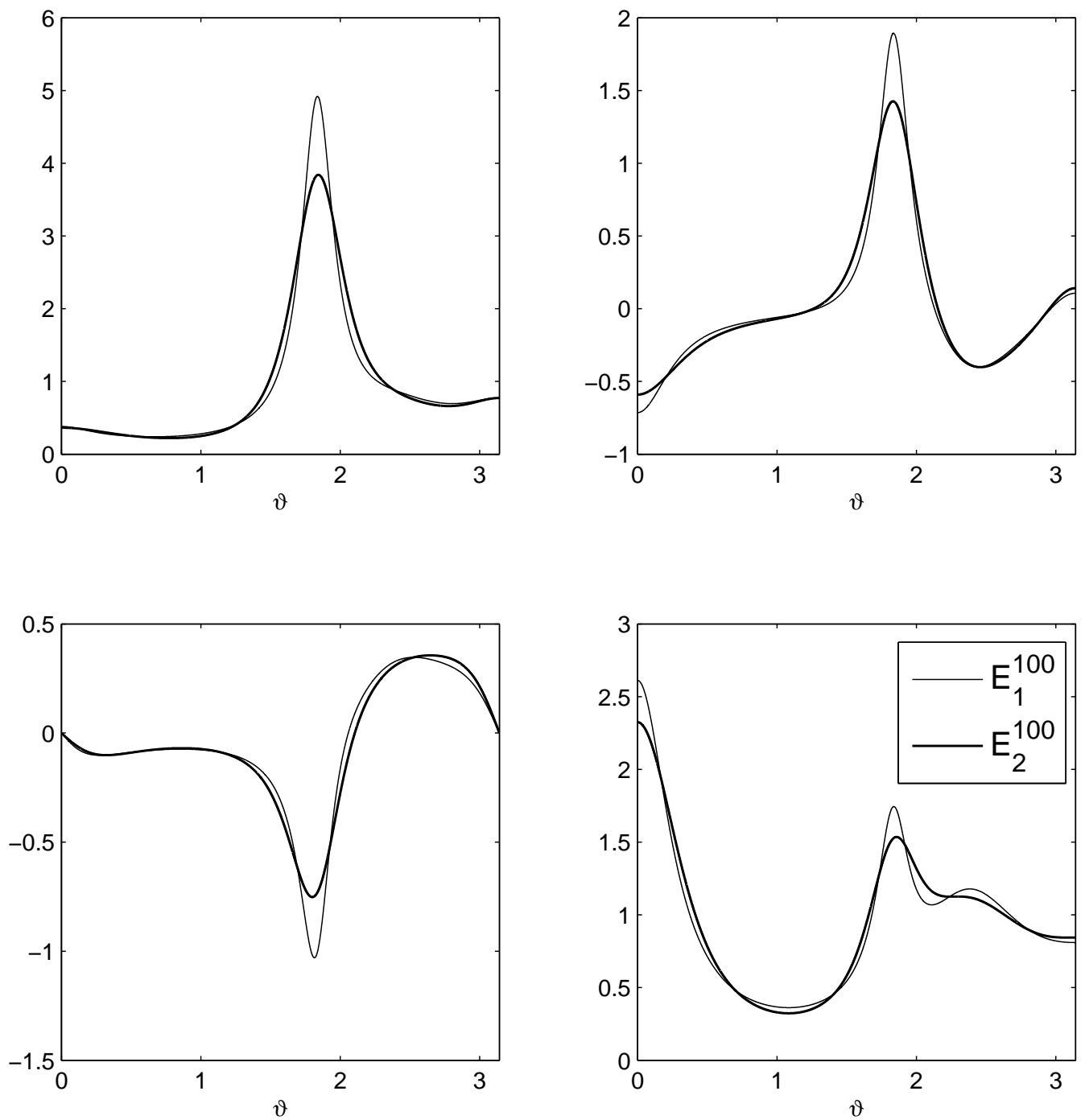

Fig. 2. Spectral densities of the normalized innovation process averaged over 50 runs with $N=100$.

similar to WGN with variance $I$ than $E_{1}^{100}$. It is also interesting to analyze the shape of $E_{\nu, k}^{N}$ changing $\nu$. For simplicity consider the scalar case, $m=1$, and let $\bar{e}=\Phi\left(e^{i \bar{\vartheta}}\right) \Psi\left(e^{i \bar{\vartheta}}\right)^{-1}$ where 
$\bar{\vartheta} \in[0,2 \pi)$ is fixed. The function

$$
f_{\nu}(\bar{e})=\bar{e}-\frac{\nu}{\nu-1}(\bar{e})^{\frac{\nu-1}{\nu}}
$$

is the infinitesimal contribution at $\bar{\vartheta}$ to the objective function $\frac{1}{\nu}\left(\mathcal{S}_{T}^{\left(1-\nu^{-1}\right)}(\Phi \| \Psi)-\frac{\nu}{\nu-1}\right)$ which is equivalent to $\mathcal{S}_{T}^{\left(1-\nu^{-1}\right)}(\Phi \| \Psi)$ in Problem 1 . Note that, $d f_{\nu}(\bar{e}) / d \bar{e}=1-e^{-\frac{1}{\nu}}$ represents the instantaneous rate of change of $f_{\nu}(\bar{e})$ at point $\bar{e}$. Moreover, $d f_{\nu_{1}}(\bar{e}) / d \bar{e} \geq d f_{\nu_{1}}(\bar{e}) / d \bar{e} \geq 0$ with $\nu_{1} \geq \nu_{2}$ and $\bar{e} \geq 1$. Accordingly the larger $\nu$ is, the more $f_{\nu}$ penalizes values of $\bar{e}$ greater than one. Therefore a sufficiently large value of $\nu$ should avoid solution whose innovation process is greater than $I$ in narrow ranges of frequencies.

We conclude that $\Phi_{\nu}$ with $\nu$ small is preferable when the model for $y$ should be simple in terms of complexity degree whereas the one with $\nu$ large is preferable when a small estimation error (also in terms of innovation process) is required. Regarding the computational complexity, the small $\nu$ is, the better performance is. In fact, to solve equation (43) it is required to construct a state space model having state dimension which is proportional to $\nu$, see [17, Section VI].

\section{CONCLUSION}

In this technical note we have presented a new multivariate divergence family between spectral densities which arises in the context of optimal prediction. Such a divergence family leads to a family of solutions to the spectrum approximation problem which are with bounded McMillan degree under the mild assumption that the a priori spectral density has bounded McMillan degree. Finally, a simulation study has been presented for drawing the application scenarios of this family of spectral estimators.

\section{REFERENCES}

[1] S. Amari. Differential-Geometrical Methods in Statistics. Springer-Verlag, Berlin, 1985.

[2] A. Basu, I. Harris, N. Hjort, and M. Jones. Robust and efficient estimation by minimising a density power divergence. Biometrika, 85(3):549-559, Sep. 1998.

[3] C. Byrnes, T. Georgiou, and A. Lindquist. A new approach to spectral estimation: A tunable high-resolution spectral estimator. IEEE Trans. Signal Processing, 48(11):3189-3205, Nov. 2000.

[4] A. Ferrante, C. Masiero, and M. Pavon. Time and spectral domain relative entropy: A new approach to multivariate spectral estimation. IEEE Trans. Autom. Control, 57(10):2561-2575, Oct. 2012.

[5] A. Ferrante, M. Pavon, and F. Ramponi. Hellinger versus Kullback-Leibler multivariable spectrum approximation. IEEE Trans. Autom. Control, 53(4):954-967, May 2008. 
[6] A. Ferrante, M. Pavon, and M. Zorzi. A maximum entropy enhancement for a family of high-resolution spectral estimators. IEEE Trans. Autom. Control, 57(2):318-329, Feb. 2012.

[7] A. Ferrante, M. Pavon, and M. Zorzi. Structured covariance estimation in high resolution spectral analysis. In Proc. of Int. Symp. Mathematical Theory of Network and Systems, MTNS 2012. Melbourne, 2012.

[8] T. Georgiou. The structure of state covariances and its relation to the power spectrum of the input. IEEE Trans. Autom. Control, 47(7):1056-1066, Jul. 2002.

[9] T. Georgiou. Relative entropy and the multivariable multidimensional moment problem. IEEE Trans. Inform. Theory, 52(3):1052-1066, Mar. 2006.

[10] T. Georgiou and A. Lindquist. Kullback-Leibler approximation of spectral density functions. IEEE Trans. Inform. Theory, 49(11):2910-2917, Nov. 2003.

[11] F. Itakura and S. Saito. Analysis synthesis telephony based on the maximum likelihood method. In Proceedings of 6th International Congress on Acoustics, pages 17-20, Tokyo, Japan, 1968.

[12] X. Jiang, L. Ning, and T. Georgiou. Distances and riemannian metrics for multivariate spectral densities. IEEE Trans. Autom. Control, 57(7):1723-1735, Jul. 2012.

[13] T. Kailath, A. Sayed, and B. Hassibi. Linear estimation. Prentice Hall, 2000.

[14] S. Kullback and R. Leibler. On information and sufficiency. The Annals of Mathematical Statistics, 22(1):79-86, 1951.

[15] A. Lindquist. Prediction-error approximation by convex optimization. In A. Chiuso, A. Ferrante, and S. Pinzoni, editors, Modeling, Estmation and Control:Festschrift in honor of Giorgio Picci on the occation of his sixty-fifth birthday, pages 265-275. Springer-Verlag, 2007.

[16] A. Lindquist and G. Picci. Linear stochastic systems: A geometric approach to modeling, estimation and identification. In preparation: preprint available in http://www.math.kth.se/ alq/LPbook

[17] F. Ramponi, A. Ferrante, and M. Pavon. A globally convergent matricial algorithm for multivariate spectral estimation. IEEE Trans. Autom. Control, 54(10):2376-2388, Oct. 2009.

[18] M. Zorzi. A new family of high-resolution multivariate spectral estimators. IEEE Trans. Autom. Control, In Press.

[19] M. Zorzi. Rational approximations of spectral densities based on the Alpha divergence. Math. Control Signals Syst., In Press.

[20] M. Zorzi and A. Ferrante. On the estimation of structured covariance matrices. Automatica, 48(9):2145-2151, Sep. 2012. 